\title{
Predicting Posttraumatic Growth among Primary Caregivers of Neonates with Hyperbilirubinemia Admitted in Neonatal Intensive Care Unit
}

\author{
Simplejit Kaur Dhanoa ${ }^{1} \&$ Manmohan Singh ${ }^{2}$ \\ ${ }^{1}$ Dept. of Psychology, Chandigarh University, India \\ ${ }^{2}$ PRC, Panjab University, Chandigarh, India \\ Correspondence: Simplejit Kaur Dhanoa, Dept. of Psychology, Chandigarh University, India.
}

Received: December 14, 2020

Accepted: February 27, 2021

Online Published: March 1, 2021

doi:10.5539/ijps.v13n1p34

URL: https://doi.org/10.5539/ijps.v13n1p34

\begin{abstract}
Preterm neonatal care is important aspect of parenting. The main objective of this study is to find out the predictors of Posttraumatic growth among primary caregivers of newborn babies affected with hyperbilirubinemia. In this study caregivers of preterm babies with hyperbilirubinemia were studied to find out the predictors of posttraumatic growth. The sample of 300 parents was collected. The results of this study were found that diverse variables such as coping skills, personality traits and parental stress are act as predictors of posttraumatic growth among parents of neonates with hyperbilirubinemia. In Conclusion, Neonate Caregivers need special psychological care as suggestions and recommendations are incorporated to prevent caregiver stress at initial stage and also need to promote mental health care professionals at antenatal and postnatal levels.
\end{abstract}

Keywords: hyperbilirubinemia, posttraumatic growth, primary caregiver, personality, parental stress, coping skills

\section{Introduction}

Birth of child is a source of happiness to parents. Unfortunately, premature infants are at risk of developmental complications. Prematurity is the leading cause of death among newborns and also evidenced by need for neonatal intensive care (Pulver, 2010; Tedeschi \& Calhoun, 1996). Birth of preterm newborns is at risk for several complications including hyperbilirubinemia and also for admission to Neonatal Intensive care units (Dani et al., 2009).

Majority of the cases of neonatal hyperbilirubinemia are related to biological causes. However, severe levels of bilirubin may lead to neurodevelopmental abnormalities like hearing loss or developmental delays (Connolly \& Volpe, 1990).

World widely 36 per cent neonates die because of infections and complicated deliveries whereas in India it has been estimated to be 52 per cent (Chawla et al., 2012). Information about the incidence of neonatal jaundice is not available in most of the developing countries because majority of deliveries occur at home (Crae \& Costa, 1999). A study from a neonatal unit in Karachi reported that the occurrence of neonatal jaundice is 13.5 per cent. Neonatal jaundice is recognized as a serious problem in other Asian countries too (Parkash \& Das, 2005).

Chronic and persistent medical conditions among children constitute consequential stressors among parents and here the parents of children with hyperbilirubinemia are no exception (Ho, 1992; Dixon et al., 2005). Neonate with hyperbilirubinemia may cause financial burden on parents (Boyd, 2002). Caring a neonate with hyperbilirubinemia have increased the levels of stress among parents (Mc Donald, Middleton, \& Dowswell, 2013).

Caregiver burnout during prolonged illness of infant is the final step in caregiver burden, where the caregivers encountered unhealthy environment while providing care. There is a wide literature about families of children with chronic illness, which have catalogued detrimental effects of the pre term ill child on the environment and functioning of the family (Boyd, 2002). However, there is paucity of studies which have explored the positive aspect. Individual as caregiver can experience positive outcomes when dealing with the severe stress and simultaneously may explore his or her existing coping skills to curb stressors. Posttraumatic growth is a positive 
psychological change or shift, due to major life trauma. A stressful situation becomes an opportunity not only to face negative feelings, but can also bring positive changes (Vinayak \& Dhanoa, 2017). Posttraumatic growth can occur after various kinds of trauma but in depth perspective has not been studied so well in parents of neonates with hyperbilirubinemia.

Many parents are not well aware about the effects of hyperbilirubinemia, but once they are aware that the high level of bilirubin can damage brain cells of the neonates (Ullah, Rahman, \& Hedayati, 2016) and can cause seizures or deafness, or serious developmental delays, then the parent's experienced trauma. The timely treatment, regular follow ups of neonates, coping efforts of parents and family support can result in some positive outcomes (Tedeschi \& Calhoun, 1996).

\subsection{Present Study}

Literature has revealed that not much work has been done on the parents of neonates with hyperbilirubinemia. Hence, this study has been designed to examine the various predictors of posttraumatic growth among the parents of neonates with hyperbilirubinemia. Posttraumatic Growth is experiencing a positive change characterized with several concepts in the literature namely: posttraumatic growth, stress conversion, positive psychological changes, positive adjustment and positive adaptation (Vinayak, Dhanoa, \& Vinayak, 2016).

\subsection{Objectives}

To examine the parental stress, personality traits and coping styles as predictors of posttraumatic growth among the mothers and fathers of male and female neonates with hyperbilirubinemia.

\subsection{Hypotheses}

It is expected that parental stress (H1a), personality (H1b) and coping styles (H1c) would predict posttraumatic growth of the mothers and fathers of male and female neonates with hyperbilirubinemia.

\section{Method}

The aim of the present investigation was to study the predictors of posttraumatic growth among parents of neonates with hyperbilirubinemia. For the present investigation, initially about 1400 parents were contacted. Out of these, the sample of total three hundred was selected based on the inclusion criteria.

\subsection{Inclusion Criteria}

1. Parents of preterm neonates having gestational age between 35 to 37 weeks with bilirubin level between $17 \mathrm{mg} / \mathrm{dl}$ to $24 \mathrm{mg} / \mathrm{dl}$.

2. Parents having first live born baby who undergoes the treatment of phototherapy for more than 24 hours.

3. Sample belongs to middle socio-economic strata with minimum educational qualification as higher secondary and from nuclear family structure.

4. Mothers were non-working.

5. Parents who were ready for 3 years follow up of their infant and also given consent in written

\subsection{Sampling Procedures}

The sample was divided into 150 fathers and 150 mothers of neonates with hyperbilirubinemia. The sample was further divided into fathers of male and female neonates with hyperbilirubinemia (i.e. 75 fathers of male neonates \& 75 fathers of female neonates) and mothers of male and female neonates with hyperbilirubinemia (i.e. 75 mothers of male neonates \& 75 mothers of female neonates). The parents in the age range of 20-35 years with first live born neonate with hyperbilirubinemia from the clinical setting were selected. Those parents whose new-born was preterm with more than $17 \mathrm{mg} / \mathrm{dl}$ bilirubin level and with educational qualification of at least higher secondary level were included. The samples were taken from the government and private hospitals of urban area as most of the patients from rural areas come to these hospitals for the treatment of hyperbilirubinemia and also for the follow-ups of the children.

\subsection{Ethical Considerations}

The ethical standards were maintained. The participants were were assured that the data collected from them will be used for research purposes and their identity would be kept confidential. Thereafter, the written consent of all the participants was taken. 


\subsection{Instruments}

The following tests were used in the present investigation:

1. Ways of Coping Inventory (WCI)(Folkman \& Lazarus, 1985)

2. NEO-Personality Inventory (Big-5)(Costa \& McCrae, 1985)

3. The Parental Stress Scale (PSS)(Berry \& Jones, 1995)

4. Posttraumatic Growth Inventory (PTGI) (Tedeschi \& Calhoun, 1996)

\subsection{Scoring}

Scoring for all the tests was done as per the instructions provided in the scoring manuals of the tests. Normality of the data was assessed before conducting the statistical analyses. Keeping in mind the objectives of the study, statistical analyses were applied i.e. step-wise multiple regression analysis.

\section{Results}

In the regression analysis for posttraumatic growth as the criterion the following variables were entered as predictors' parental stress, personality (viz. openness, conscientiousness, agreeableness, extraversion and neuroticism), ways of coping (viz. social isolation, social support, self-blame, empathy, distancing, tension reduction, problem solving).

Among mothers of male and female neonates with hyperbilirubinemia, personality viz. openness and extraversion variables turned out to be relevant and were retained as predictors as they explained $46 \%\left(R^{2}=0.45\right)$ of the variance in the criterion variable i.e. posttraumatic growth. The predictors which emerged significant were openness $(\beta=0.46)$, extraversion $(\beta=0.40)$. Among fathers of male and female neonates with hyperbilirubinemia, ways of coping viz. self-blame and social isolation variables turned out to be relevant and were retained as predictors as they explained $63 \%\left(\mathrm{R}^{2}=0.63\right)$ of the variance in the criterion variable i.e. posttraumatic growth. The predictors which emerged significant were self-blame $(\beta=0.27)$, social isolation $(\beta=0.11)$.

Table 1. Step wise- Regression Equations for PTG on various groups

\begin{tabular}{|c|c|c|c|c|c|c|}
\hline Groups & Predictor Variables & Std. Co-efficient $\beta$ & $\mathbf{t}$ & $\overline{\mathbf{R}^{2}}$ & $\mathbf{R}^{2}$ Change & F Change \\
\hline \multirow[t]{2}{*}{$\mathbf{M N}$} & $\mathrm{O}$ & 0.46 & $5.31 * *$ & 0.28 & 0.05 & $10.99 * *$ \\
\hline & $\mathrm{E}$ & 0.4 & $4.38 * *$ & 0.35 & 0.07 & $15.88 * *$ \\
\hline \multirow[t]{3}{*}{$\mathbf{F N}$} & SB & 0.27 & $3.48 * *$ & 0.58 & 0.13 & $47.10 * *$ \\
\hline & $\mathrm{CON}$ & 0.14 & $2.75 * *$ & 0.61 & 0.02 & $8.73 * *$ \\
\hline & SI & 0.11 & $2.15^{*}$ & 0.63 & 0.01 & $4.64 * *$ \\
\hline \multirow[t]{2}{*}{ MFN } & $\mathrm{O}$ & 0.22 & $2.71 * *$ & 0.48 & 0.05 & $7.53 * *$ \\
\hline & EM & 0.17 & $2.13 * *$ & 0.56 & 0.02 & $4.53 * *$ \\
\hline \multirow[t]{4}{*}{ FFN } & SI & 0.22 & $6.09 * *$ & 0.32 & 0.08 & $40.21 * *$ \\
\hline & $\mathrm{O}$ & 0.03 & $0.40 *$ & 0.49 & 0.07 & $10.15 * *$ \\
\hline & PBS & 0.25 & $3.61 * *$ & 0.53 & 0.04 & $6.26^{* *}$ \\
\hline & A & 0.37 & $4.86^{* *}$ & 0.58 & 0.05 & $8.66^{* *}$ \\
\hline \multirow[t]{2}{*}{ MMN } & PBS & 0.22 & $2.25 *$ & 0.26 & 0.05 & $5.16 * *$ \\
\hline & PS & 0.23 & $3.67 * *$ & 0.49 & 0.02 & $7.16^{* *}$ \\
\hline \multirow[t]{4}{*}{ FMN } & $\mathrm{SS}$ & 0.47 & $4.58 * *$ & 0.18 & 0.18 & $16.30 * *$ \\
\hline & SI & 0.22 & $2.31 * *$ & 0.15 & 0.09 & $12.01 * *$ \\
\hline & A & 0.25 & $2.48 * *$ & 0.24 & 0.06 & $6.18 * *$ \\
\hline & PS & 0.33 & $4.76^{* *}$ & 0.28 & 0.26 & $8.84 * *$ \\
\hline
\end{tabular}

${ }^{*} \mathbf{p} \leq \mathbf{0 . 0 5},{ }^{* *} \mathbf{p} \leq \mathbf{0 . 0 1}$

PTG= Posttraumatic Growth, PS= Parental Stress, $\mathrm{O}=$ Openness, $\mathrm{E}=\mathbf{E x t r a v e r s i o n , ~} \mathrm{A}=\mathrm{Agreeableness,}$ $\mathbf{C}=$ Conscientiousness, $\mathbf{N}=$ Neuroticism, $\mathbf{S I}=$ Social Isolation, $\mathbf{S B}=$ Self Blame, $\mathbf{D}=\mathbf{D i s t a n c i n g}, \mathbf{I N D}=$ Independent, IC = Intellectual-Cultural, $\mathbf{P B S}=$ Problem solving, EM= Empathy; SS=Social; Support. $\mathrm{MN}=$ Mothers of neonates, $\mathrm{FN}=$ Fathers of neonates, MFN= Mothers of Female neonates, FFN= Fathers of Female neonates, $M M N=$ Mothers of Male neonates, FMN= Fathers of Male neonates. 
Among mothers of male neonates with hyperbilirubinemia, personality viz. openness and ways of coping viz. empathy variables tuned out to be relevant and were retained as predictors as they explained $56 \%\left(\mathrm{R}^{2}=0.56\right)$ of the variance in the criterion variable i.e. posttraumatic growth. The predictors which emerged significant were problem solving $(\beta=0.22)$, openness $(\beta=0.19)$ and empathy $(\beta=0.17)$. Among fathers of male neonates with hyperbilirubinemia, parental stress, personality viz. agreeableness, openness variables tuned out to be relevant and were retained as predictors as they explained $68 \%\left(\mathrm{R}^{2}=0.68\right)$ of the variance in the criterion variable i.e. posttraumatic growth. The predictors which emerged significant were agreeableness $(\beta=0.37)$, openness $(\beta=$ $0.03)$.

Among mothers of female neonates with hyperbilirubinemia, ways of coping viz. problem solving were retained as predictors as they explained $31 \%\left(\mathrm{R}^{2}=0.31\right)$ of the variance in the criterion variable i.e. posttraumatic growth. The predictors which emerged significant were expressiveness $(\beta=0.40)$, moral religious $(\beta=0.22)$. Among fathers of female neonates with hyperbilirubinemia, ways of coping viz. social support, personality viz. agreeableness were retained as predictors as they explained $25 \%\left(\mathrm{R}^{2}=0.24\right)$ of the variance in the criterion variable i.e. posttraumatic growth. The predictors which emerged significant were social support $(\beta=0.47)$, agreeableness $(\beta=0.25)$, problem solving $(\beta=0.25)$.

\section{Discussion}

The objective of the study was to find out the significant predictors for criterion variable i.e. posttraumatic growth. For this, step-wise multiple regression analysis was applied. The variables i.e. parental stress, personality and ways of coping and their dimensions were entered as predictors. Posttraumatic growth (PTG) is the used to construct the positive experiences with the help of psychological and cognitive efforts to deal with challenging circumstances (Connolly \& Volpe, 1990).

\subsection{Parental Stress}

Caregivers of ill children reported that stress, growth and the duration of illness, worries about developmental delays in child (Vinayak \& Dhanoa, 2017). In order to gauge the role of parental stress in predicting posttraumatic growth, hypotheses (H1a) was framed which was expected parental stress as a predictor of posttraumatic growth among parents of neonates with hyperbilirubinemia. Only two group i.e. mothers and fathers of male neonate's revealed parental stress as a predictor of posttraumatic growth. The precise reason behind the results is based on PTG model (Parkash \& Das, 2005), they explained that three environmental factors that have been associated with perceived growth are gender, age and educational level. Parents have been found to be psychologically affected by their child's diagnosis and this stress may predict posttraumatic growth. Studies supported the results and decipher that parental stress have proven it as a significant predictor of posttraumatic growth.

Another reason could be the socio-cultural perspectives. In north region of India, preference for male child is higher as compared to female child. Especially, the birth of first male child is considered as auspicious for the families (Dennery, Seidman, \& Stevenson, 2001). The diagnosis of jaundice among male neonates was preferably the common cause of stress among their parents and the treatment for illness of children are often associated with posttraumatic growth (Berry \& Jones, 1995). Triggering event shakes the person's dream world and plays a key role in predicting the degree of posttraumatic growth (Dixon et al., 2001).

\subsection{Personality and Its Dimensions}

Studies suggested that the BIG five characteristics "extraversion", "openness to experience", "agreeableness" and "conscientiousness" have a positive relation with PTG, and also predict the PTG (Dennery, Seidman, \& Stevenson, 2001). In this present study, openness was emerged as a predictor of PTG in mothers of male and female neonates, fathers of female neonates. The hypothesis $(\mathrm{H} 1 \mathrm{~b})$ was supported with detailed reasons that openness has more modest relationships with PTG than other personality traits (Costa \& McCrae, 1985). Posttraumatic growth influenced with the degree to which an individual perceived their own change. The another reason for openness as predictor of PTG among mothers and fathers of male neonates is supported and revealed by few studies (Vinayak, Dhanoa, \& Vinayak, 2016) that individuals who were exposed to real world traumatic events shown major personality characteristics of five factor models and are more related to reaction to trauma.

The other personality trait is extraversion and it tends to be enthusiastic, action-oriented individuals. In the present study, posttraumatic growth is significantly predicted by extraversion (H1b) in mothers of male and female neonates. The distinct reason in support of results is that extraversions are expected to engage with social support. The mothers of male and female neonates with hyperbilirubinemia easily engage with the mothers of other neonates admitted in NICU and they have shared information which may help them to attain positive 
growth.

In this study, agreeableness was act as predictor of PTG in fathers of male and female neonates. The results supported the hypothesis (H1b) with evidence that agreeableness trait (i.e. five factor model: (Pulver et al., 2010) may also play an important role in reaction to traumatic events.

\subsection{Ways of Coping and Its Dimensions}

The type of coping style used immediately after the trauma is associated with cognitive processes. The self-blame is a coping which may explain the situational meaning of an event. The findings of the present study also supported the hypothesis (H1c) with self-blame as predictor of PTG among fathers of male and female neonates. The explicit reason in support of result is that in Indian culture, head of the family is liable for financial arrangements. The single earner in family often comes under stress and use various defense mechanism to deal with newborn. During the hospitalization of newborns, the fathers as compared to mothers sometimes blame themselves for the illness of their newborns. Few studies supported that self-blaming predict positive growth among fathers as compare to mothers (Dani et al., 2009).

The social isolation is another coping process which explained lack of contact between an individual and community. In this present study, social isolation emerged as a significant predictor for posttraumatic growth among the fathers of male and female neonates. The comprehensive reason for this could be the situations and traumatic experiences influenced the individual to use different coping skills (Dennery, Seidman, \& Stevenson, 2001).

Problem solving as coping mechanism also emerged as predictor of PTG among fathers of female neonates and mothers of male neonates. The hypothesis (H1c) supported with the rationale that problem solving is inter-linked with personality and with social support. All these factors contribute towards PTG. In this present study, all these characteristics were existed in fathers of female and mothers of male to predict the posttraumatic growth. Studies also supported the results with reason that caregivers who have cohesive environment in family and have intellectual abilities may use problem solving strategies and are more prone towards PTG (WHO, 2016).

\subsection{Implications and Suggestions}

Several attempts to strengthen newborn care in India have been made, but a review of these interventions revealed that their overall impact on neonatal mortality was limited. High bilirubin may cause neurological impairment even in term newborns. Caring a neonate in NICU is extremely stressful and this research gives an insight into posttraumatic growth among parents of neonates with hyperbilirubinemia and its predictors. Better understanding and insight can surely help us to ascertain interventions and coping strategies to reduce the effect of traumatic events. In India, there is a need for additional education regarding the care needed and the effects of neonatal illness on neonates as well as the caregivers. This research is the first of its kind in India, to use a large sample to quantitatively document the experiences of the mothers and fathers of male and female neonates with hyperbilirubinemia and attempted to find predictors of posttraumatic growth.

The present study suggested that that parent often experienced a range of emotions as anxiety, confusion after knowing their child's illness. The first experience of having a first child with illness is very traumatic but the family support system and coping skills of individuals and persons own personality traits may help them to waive with such situations. Early discharge without follow-up, low maternal knowledge about caring a neonate, cultural practices and use of traditional treatments may limit or delay detection or care-seeking for jaundice. An education campaign to modify community care practices and traditional beliefs may improve care-seeking and caregiving and providing laboratory and phototherapy capabilities at hospitals could improve detection and treatment of hyperbilirubinemia. The evidence-based programs must be developed and separate counseling cells with neonatal psychologist, should be set up to train, help and counsel the parents.

\subsection{Conclusion}

Based on the findings of the present study, it is clear that caregivers of neonates experienced posttraumatic growth if supported with coping skills. There is a crucial need to develop different coping strategies which respond positively to individual's changing needs, especially for the parents of neonates admitted in NICU. In conclusion, this study contributes to an improved understanding of how certain psychological factors can influence the health of parents as primary caregiver.

\section{References}

Berry, J. O., \& Jones, W. H. (1995). The parental stress scale: Initial psychometric evidence. Journal of Social Personality Relationships, 12, 463-472. https://doi.org/10.1177/0265407595123009 
Boyd, B. A. (2002). Examining the relationship between stress and lack of social support in mothers of children with autism. Journal Autism Other Developmental Disabilities, 17(4), 208-215. https://doi.org/10.1177/10883576020170040301

Chawla, D., Jain, S., Dhir, S., \& Rani, S. (2012). Risk assessment strategy for prediction of pathological hyperbilirubinemia in neonates. Indian Journal of Pediatrics, 79, 198-201. https://doi.org/10.1007/s12098-011-0409-x

Connolly, A. M., \& Volpe, J. J. (1990). Clinical features of bilirubin encephalopathy. Clinics in Perinatology, 17(2), 371-379. https://doi.org/10.1016/S0095-5108(18)30573-6

Costa, P. T., \& McCrae, R. R. (1985). The NEO Personality Inventory manual. Psychological Assessment Resource, 3, 12-16. https://doi.org/10.1037/t07564-000

Dani, C., Corsini, I., \& Piergentili, L. (2009). Neonatal morbidity in late preterm and term infants in the nursery of a tertiary hospital. Acta of Pediatrics, 98(11), 1841-1843. https://doi.org/10.1111/j.1651-2227.2009.01425.x

Dennery, P. A., Seidman, D. S., \& Stevenson, D. K. (2001). Neonatal hyperbilirubinemia. New England Journal of Medicine, 344(8), 581-590. https://doi.org/10.1056/NEJM200102223440807

Dixon-Woods, M., Young, B., \& Heney, D. (2005). Rethinking experiences of childhood cancer: A multidisciplinary approach to chronic childhood illness: a multidisciplinary approach to chronic childhood illness. McGraw-Hill Education (UK).

Ho, N. K. (1992). Neonatal jaundice in Asia. Baillieres Clinical Haematology, 5(1), 131-142. https://doi.org/10.1016/S0950-3536(11)80038-7

Crae, R. R., \& Costa, P. T. (1999). A five-factor theory of personality. Handbook of Personality: Theory and Research, 2, 139-153.

McDonald, S. J, Middleton, P., \& Dowswell, T. (2013). Effect of timing of umbilical cord clamping of term infants on maternal and neonatal outcomes. Cochrane Database of Systematic Reviews, 7, 12-18. https://doi.org/10.1002/14651858.CD004074.pub3

Parkash, J., \& Das, N. (2005). Pattern of admissions to neonatal unit. Journal of College of Physic Surgeons, 15(6), 341-344.

Pulver, L. S., Denney, J. M., \& Silver, R. M. (2010). Morbidity and discharge timing of late preterm newborns. Clinical of Pediatrics, 49(11), 1061-1067. https://doi.org/10.1177/0009922810376821

Tedeschi, R. G., \& Calhoun, L. G. (1996). The Posttraumatic Growth Inventory: Measuring the positive legacy of trauma. Journal of Traumatic Stress, 9(3), 455-471. https://doi.org/10.1007/BF02103658

Ullah, S., Rahman, K., \& Hedayati, M. (2016). Hyperbilirubinemia in Neonates: Types, Causes, Clinical Examinations, Preventive Measures and Treatments: A Narrative Review Article. International Journal of Public Health, 45(5), 558.

Vinayak, S., \& Dhanoa, S. K. (2017). Relationship of parental burnout with parental stress and personality among parents of neonates with hyperbilirubinemia. International Journal of Indian Psychology, 4(2), 102-111.

Vinayak, S., Dhanoa, S. K., \& Vinayak, R. (2016). Relationship of hopelessness, depression and quality of life in mothers of persons with disabilities. International Journal of Innovative Applied Studies, 17(1), 306.

World Health Organization. (2016). Towards a grand convergence for child survival and health: a strategic review of options for the future building on lessons learnt from IMNCI. Retrieved from https://apps.who.int/iris/handle/10665/251855

\section{Copyrights}

Copyright for this article is retained by the author(s), with first publication rights granted to the journal.

This is an open-access article distributed under the terms and conditions of the Creative Commons Attribution license (http://creativecommons.org/licenses/by/4.0/). 